\title{
Atypical anti-glomerular basement membrane glomerulonephritis in a patient with metastatic melanoma treated with mitogen-activated protein kinase and immune checkpoint inhibitors: a case report
}

Periklis Kyriazis ${ }^{1 *}$, Abhinav Tiwary², Jonathan Freeman ${ }^{3}$, Daniel Landry ${ }^{2}$ and Gregory Braden ${ }^{2}$

\begin{abstract}
Background: Immune checkpoint inhibitors and mitogen-activated protein kinase inhibitors have become the standard of care in patients with advanced melanoma bearing V600 mutations. However, little is known about their nephrotoxicity. To date, only two cases of anti-glomerular basement membrane glomerulonephritis after exposure to checkpoint inhibitors have been documented. Herein, we report the first case of a patient with metastatic melanoma who developed linear Immunoglobulin G 3+, Immunoglobulin A 2+, kappa 2+, lambda 1+ anti-glomerular basement membrane glomerulonephritis with negative serology following treatment with checkpoint inhibitors and subsequently mitogen-activated protein kinase inhibitors.
\end{abstract}

Case presentation: A 58-year-old Caucasian male was referred to our outpatient nephrology clinic with acute kidney injury and proteinuria. He had received three cycles of ipilimumab and nivolumab for recurrent melanoma positive for the BRAF V600E mutation with metastasis to the lungs. Immunotherapy had been discontinued in the setting of severe adverse effects including dermatitis, colitis, and hepatitis. Because of persistent bilateral lung metastases and left pleural metastases, the patient had been initiated on dabrafenib and trametinib until his presentation to our clinic 6 months later. On presentation, his blood pressure was 172/89 mm/Hg and had 2+ edema bilaterally. His creatinine level was $2.4 \mathrm{mg} / \mathrm{dL}$ from a previous normal baseline with a urinary protein-to-creatinine ratio of $2 \mathrm{~g} / \mathrm{g}$. His urinalysis showed dysmorphic erythrocytes and red blood cell casts. Serologic testing was negative for antineutrophilic cytoplasmic antibodies, proteinase 3 antigen, myeloperoxidase, and anti-glomerular basement membrane antibody. Complement levels were normal. A renal biopsy showed focal crescentic (2 of 15 glomeruli with cellular crescents), proliferative, and sclerosing glomerulonephritis with diffuse linear staining of glomerular capillary loops dominant for $\lg G(3+), \lg A(2+)$, kappa $(2+)$, and lambda $(1+)$ minimal changes. He was initiated on oral cyclophosphamide and

*Correspondence: pkyriazi@bidmc.harvard.edu

${ }^{1}$ Dept. of Internal Medicine, University of Massachusetts Medical School

-Baystate, Springfield, MA, USA

Full list of author information is available at the end of the article

(c) The Author(s) 2021. Open Access This article is licensed under a Creative Commons Attribution 4.0 International License, which permits use, sharing, adaptation, distribution and reproduction in any medium or format, as long as you give appropriate credit to the original author(s) and the source, provide a link to the Creative Commons licence, and indicate if changes were made. The images or other third party material in this article are included in the article's Creative Commons licence, unless indicated otherwise in a credit line to the material. If material is not included in the article's Creative Commons licence and your intended use is not permitted by statutory regulation or exceeds the permitted use, you will need to obtain permission directly from the copyright holder. To view a copy of this licence, visit http://creativecommons.org/licenses/by/4.0/. The Creative Commons Public Domain Dedication waiver (http://creativeco mmons.org/publicdomain/zero/1.0/) applies to the data made available in this article, unless otherwise stated in a credit line to the data. 
pulse intravenous methylprednisolone followed by oral prednisone for 6 months, which stabilized his renal function until reinitiation of immunotherapy.

Conclusions: Acute kidney injury is an increasingly reported adverse effect of both drug classes, mostly affecting the tubulointerstitial compartment and infrequently the glomerulus. Although the biologic effect of these drugs on immune cells is not entirely understood, it is possible that BRAF-induced podocyte injury in combination with direct T-cell-mediated glomerular injury facilitated by checkpoint inhibitors led to the unmasking of cryptic antigens, loss of self-tolerance, and autoimmunity. More importantly, we show that treatment with corticosteroids and cyclophosphamide was able to improve and stabilize our patient's renal function until the reinitiation of immunotherapy.

Keywords: Atypical anti-GBM, Immune checkpoint inhibitors, BRAF inhibitors, MEK inhibitors

\section{Introduction}

Metastatic melanoma is almost invariably incurable, with a median survival time of less than 1 year and a 3-year survival rate of only $10-15 \%$.[1] The molecular pathogenesis is strongly correlated with constitutive activation of the mitogen-activated protein kinase (MAPK) (RASBRAF-MEK-ERK) pathway that excessively signals cells to grow. Melanoma proliferation is attributed to targetable activating BRAF mutations in $50 \%$ of cases.[2] In 2014, a combined treatment with dabrafenib, a selective BRAF inhibitor (BRAFi), and trametinib, a selective MEK inhibitor (MEKi) was approved by the Food and Drug Administration (FDA) for the treatment of metastatic melanoma. In 2015, a combination of checkpoint inhibitors (CPI), namely of nivolumab, a monoclonal antibody against programmed cell death receptor-1 (PD-1), and ipilimumab, a monoclonal antibody targeting cytotoxic T-lymphocyte antigen 4 (CTLA-4), was also approved for the same purpose.[3] PD-1 and CTLA-4 are part of a coinhibitory pathway at the CD28 "checkpoint" of T-cell activation. They exist to safeguard T-cell tolerance and protect against autoimmunity. In the setting of malignancy, anti-programmed cell death protein ligand 1 (PD-L1) binds to PD-1, enabling cancer cells to evade host immune surveillance.[4] Anti-CTLA-4 and anti-PD1 checkpoint blockade therapies target distinct tumor-infiltrating T-cell populations to induce tumor rejection.[5]

Despite important clinical benefits, these novel therapies are associated with nephrotoxicity.

BRAF inhibition clinically affects mostly the tubulointerstitial compartment, despite the fact that in murine studies BRAF was shown to be expressed and localized in developing and mature podocytes. Based on published case series, BRAFi can cause acute allergic interstitial nephritis within the first $1-2$ weeks or a more subacute tubular injury through direct toxicity within the first 1-2 months of initiation.[6] BRAFi is also implicated in diffuse podocyte injury through BRAF-PCLE1 interactions and nephrin downregulation, leading to nephrotic syndrome.[7]

Similarly, CPI are associated with renal injury, primarily acute interstitial nephritis (AIN) but also glomerulonephropathies, including membranous nephropathy, minimal change disease, focal segmental glomerulosclerosis (FSGS), and granulomatosis with polyangiitis and lupus nephritis. These side effects can take up to $10-12$ months to manifest. [8]

Herein, we report the first case of a patient with metastatic melanoma who developed linear Immunoglobulin (Ig) G 3+, IgA 2+, kappa 2+, lambda 1+ anti-glomerular basement membrane glomerulonephritis (anti-GBM $\mathrm{GN}$ ) with negative serology following treatment with CPI and subsequently MAPKi.

\section{Case presentation}

A 58-year-old Caucasian male presented with acute kidney injury (AKI) and proteinuria in June 2016. He had originally been diagnosed with melanoma along the right flank in the 1980s that was treated with wide excisional removal. He was followed closely for over three decades with no signs of metastatic disease until he was noted to have a mass near his prior melanoma excisional scar in summer 2014. A biopsy at that time revealed an epithelioid neoplasm with morphology and phenotype highly suggestive of melanoma that was positive for the BRAF V600E mutation. A Positron Emission Tomography/ Computed Tomography (PET/CT) at that time showed numerous bilateral pulmonary nodules, and a subsequent right upper lobe wedge resection did confirm metastatic melanoma. In May 2015, he was initiated on ipilimumab $(3 \mathrm{mg} / \mathrm{kg})$ and nivolumab $(1 \mathrm{mg} / \mathrm{kg})$ every 3 weeks for management of his metastatic melanoma. His course was complicated by grade 3 dermatitis, colitis, and hepatitis, which were treated with courses of prednisone. Therapy was ultimately discontinued in October 2015, after receiving a total of three cycles intermittently, as a result of worsening hepatitis confirmed by liver biopsy. Because of persistent bilateral lung metastases and left pleural metastases, the patient was initiated on dabrafenib $150 \mathrm{mg}$ twice daily and trametinib $2 \mathrm{mg}$ twice daily in December 2015, which he remained on until his presentation to nephrology in June of 2016. He had no personal or family history of chronic kidney disease (CKD). He denied exposure to 
nephrotoxic agents. He was only taking amlodipine $10 \mathrm{mg}$ for hypertension and had been receiving immunotherapy with dabrafenib and trametinib since December 2015. He denied tobacco use, illicit drug use, and toxic environmental exposure. His blood pressure was $172 / 89 \mathrm{~mm} / \mathrm{Hg}$ and had $2+$ edema bilaterally. The remainder of his physical examination was normal, and laboratory results indicated a creatinine level of $2.4 \mathrm{mg} / \mathrm{dL}$ with a urinary protein-tocreatinine ratio of $2 \mathrm{~g} / \mathrm{g}$. His urinalysis showed dysmorphic erythrocytes and red blood cell casts. Renal ultrasonography was normal. Serologic testing was negative for Antineutrophil cytoplasmic autoantibody (ANCA), PR-3, Myeloperoxidase (MPO), and Anti-glomerular basement membrane (anti-GBM) antibody. Complement levels were normal. A renal biopsy was performed in September of
2016 showing focal crescentic ( 2 of 15 glomeruli with cellular crescents), proliferative, and sclerosing glomerulonephritis with diffuse linear staining of glomerular capillary loops dominant for IgG (3+), IgA (2+), kappa $(2+)$, and lambda (1+) minimal changes (Fig. 1). Ultrastructural examination of three glomeruli demonstrated areas with open capillary loops and preserved foot processes. Other areas demonstrated diffuse effacement of foot processes with variable thickening and wrinkling of glomerular basement membranes. No immune complex disease or tubuloreticular structures were identified.

Repeat anti-GBM testing remained negative, and the patient's creatinine eventually rose to a peak of $3.8^{\prime} \mathrm{mg} /$ $\mathrm{dL}$. He had no signs or symptoms of lung hemorrhage. Dabrafenib and trametinib were discontinued, and he
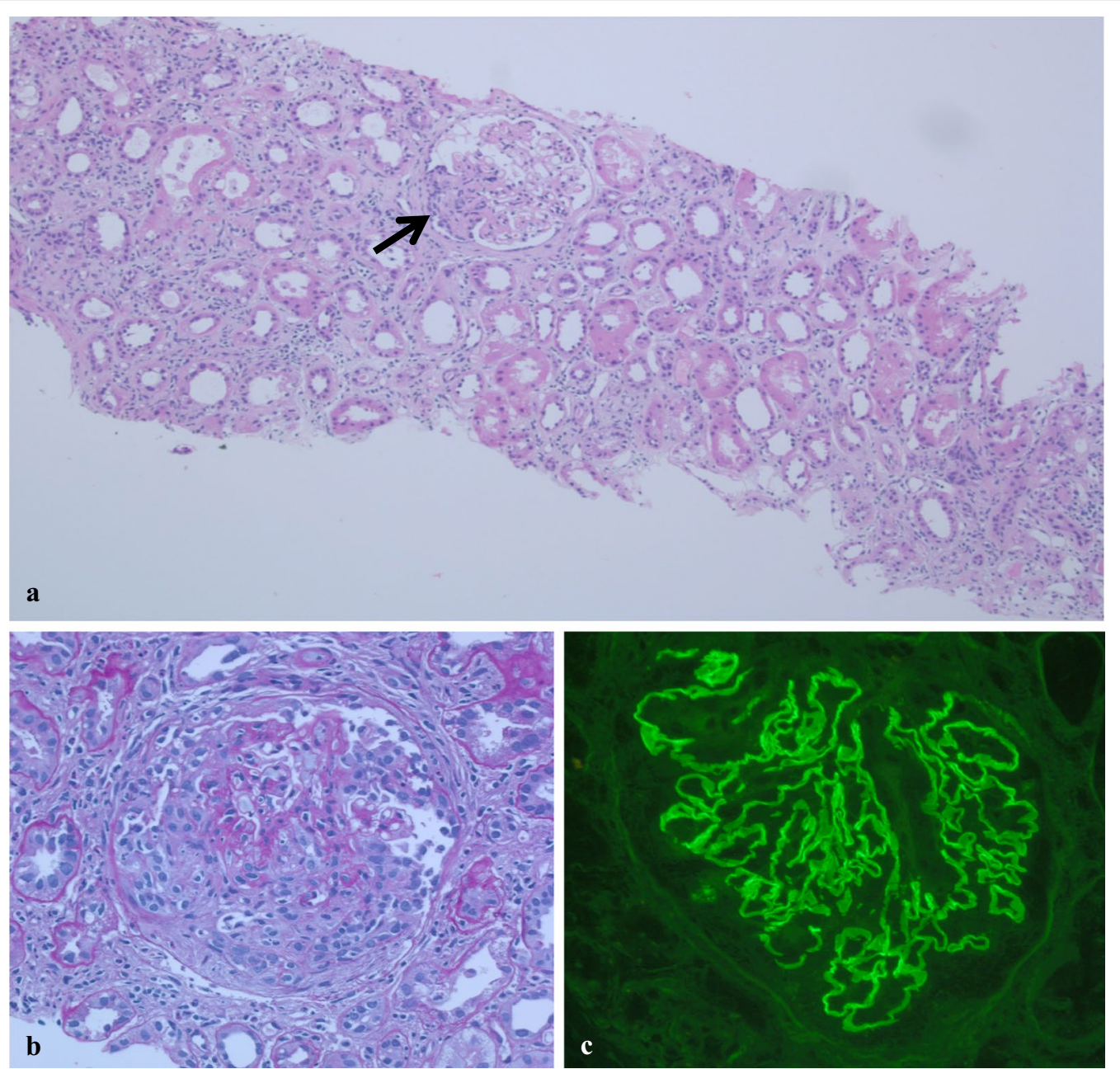

Fig. 1 a Chronic active interstitial nephritis with focal cellular crescents and linear lgG immunoglobulin deposition. Widespread acute tubular injury with luminal dilatation and flattened epithelium. A glomerulus demonstrates segmental sclerosis and capsular adhesion, possibly and previous crescent (arrow) (hematoxylin and eosin [H\&E], original magnification 40x). b Glomerulus with cellular crescent (periodic acid-Schiff [PAS], original magnification 200x). c Immunofluorescence microscopy demonstrates linear staining for Immunoglobulin G along peripheral capillary loops of all glomeruli (original magnification 200x), also for positive linear lgG3+, IgA 2+, Kappa 2+, Lambda 2 anti-glomerular basement membrane glomerulonephritis (not shown) 
was subsequently initiated on oral cyclophosphamide ( $2 \mathrm{mg} / \mathrm{kg} /$ day) and pulse intravenous methylprednisolone (1000 mg daily for 3 consecutive days) followed by $1 \mathrm{mg} / \mathrm{kg} /$ day of prednisone. Serum creatinine improved to $2.5 \mathrm{mg} / \mathrm{dL}$, and the active urinary sediment resolved. Immunosuppression with cyclophosphamide was discontinued after 4 months of therapy, and he was weaned off prednisone by 6 months.

The patient was off all immunotherapy for his malignancy, and his renal function remained relatively stable over the ensuing 12 months. By June 2018, a PET/CT of the chest showed evidence of metastatic melanoma to the left upper and medial lobes of the lung. The patient was placed back on nivolumab, which seemed to stabilize his oncologic disease. Unfortunately, 4 weeks after reinitiating nivolumab, his creatinine jumped from 2.8 to $5.8 \mathrm{mg} / \mathrm{dL}$ and home hemodialysis was initiated. The patient remains stable on home hemodialysis and was more recently taken off nivolumab and placed back on dabrafenib and trametinib for progressive metastatic pulmonary disease, which has led to significant symptom control.

\section{Discussion}

Acute glomerulonephritis due to anti-GBM antibody is a rare cause of $\mathrm{GN}$, with an incidence of 1:1,000,000, but it also is one of the most aggressive forms of GN. Autoreactive $B$ cells produce antibodies against self-antigens, which in native GBM are sequestered within the quaternary structure of the noncollagenous domains of the triple helix of alpha 3,4,5 chains.[9] Subsequently, glomerular injury occurs through complement activation by the classical pathway, IgG Fc receptor $(\mathrm{Fc} \gamma \mathrm{R})$ mediated phagocytosis or by direct T-cell-mediated toxicity.[10] Anti GBM-GN typically presents with rapidly progressive glomerulonephritis with or without pulmonary hemorrhage. Its histopathologic hallmark is linear GBM staining for IgG by immunofluorescence (IF) and diffuse crescentic/necrotizing GN affecting more than $50 \%$ of glomeruli on light microscopy. Atypical and rare presentation have also been reported. Nasr et al. [11] in a case series described 20 patients with atypical antiGBM GN that had a milder, more indolent progressive renal impairment with linear IgG deposition on kidney biopsy, without predominant features of crescentic GN ( $<40 \%$ glomeruli affected) or overt lung hemorrhage. Circulating anti-GBM antibodies were not detected using conventional assays, for which multiple explanations have been proposed, including low-affinity antibodies to the pathogenic substrate, disappearance of circulating antibodies before the resolution of the disease, and cellmediated rather than humoral immune response.[12]
There are only two cases of typical anti-GBM disease associated with CPI reported so far.[13, 14] The proximate cause for anti-GBM GN in these cases is not yet fully understood, but it is likely that CPI swung the pendulum toward a proinflammatory state conducive to autoimmunity. Specifically, it has been shown that CTL4 blockade depletes Treg populations while enhancing antitumor immunity through the expansion of tumor-infiltrating CD8 T and Th1-like CD4 effector cell populations.[15-17] Persistent antigen stimulation may eventually lead to CD8 cell exhaustion partly due to overexpression of inhibitory receptors such as PD-1, an effect that is abrogated by CPI allowing for reinvigoration of T-cell mechanisms.[5]

Further evidence supporting a link between CPI therapy and anti-GBM disease comes from animal studies. Reynolds et al. showed that CD28-B7 checkpoint blockade with an agonistic monoclonal antibody, CTLA4-Ig, that selectively binds to B7.1, reduced autoantibody production and cellular infiltration of glomeruli in an experimental autoimmune rat model of Goodpasture's disease. [18] Kitching and colleagues further corroborated these findings with an elegant murine study, showing that in humorally mediated anti-GBM GN induced by a foreign antigen, limiting antibody-mediated injury using CTLA4-Fc treatment can attenuate anti-GBM GN.[19]

GN can also be caused by antigen-specific T cells without secreted autoantibodies in experimental anti-GBM nephritis, in which the transfer of activated a3NC1specific CD4 $\mathrm{T}$ cells to naive rats caused crescentic glomerulonephritis.[20] Autoreactive T cells can subsequently prompt autoreactive B cells, which present the same T-cell epitope of endogenous autoantigens during B-cell epitope spreading, to mount a humoral response. T-cell-mediated glomerular injury may also trigger de novo internal immunization of autoantigens released from damaged GBM, which further leads to activation of a group of GBM-specific B cells.[21] Antigen dissemination could have occurred through BRAFi-induced podocyte injury since podocytes are solely responsible for the synthesis and release of the collagen isoform a3a4a5 (type IV) in mature GBM. Perico et al. in 2017 reported a case of podocyte injury presenting as nephrotic syndrome after combinatorial treatment with dabrafenib and trametinib. They also demonstrated in in vitro experiments that mainly dabrafenib caused profound slit diaphragm disassembly by impairing the BRAF-PLC 1 interactions in podocytes, leading to nephrin downregulation and increased glomerular permeability. Diffuse loss of podocyte cytoarchitecture caused extensive foot process effacement and endothelial injury.[7, 22] 


\section{Conclusions}

Taken together, we believe that the use of immunemodulating drugs created a proinflammatory milieu that, in combination with GBM injury and antigen dissemination, provoked loss of peripheral tolerance and autoimmunity. Oncologists and nephrologists should be cognizant of the association of CPI and MAPKi with antiGBM GN since their use is on the rise. Despite the availability of general guidelines in dealing with anti-GBM GN, further research establishing more specific guidelines is necessary. According to the latest KDIGO guidelines for anti-GBM GN, triple therapy with cyclophosphamide, prednisone, and plasmapheresis is recommended. Kidney transplantation for suitable candidates may be considered after circulating antibodies remain undetectable for a minimum of 6 months. In our case, discontinuation of all immunotherapy agents and treatment with prednisone and cyclophosphamide stabilized our patient's renal function, until reinitiation of nivolumab caused rapid loss of GFR and dependence on renal dialysis.

\begin{abstract}
Abbreviations
ANCA: Antineutrophilic cytoplasmic antibodies; Anti-GBM GN: Anti-glomerular basement membrane glomerulonephritis; CKD: Chronic kidney disease; CPI: Checkpoint inhibitors; CTLA-4: Cytotoxic T-lymphocyte antigen 4; FSGS: Focal segmental glomerulosclerosis; GN: Glomerulonephritis; KDIGO: Kidney disease improving global outcomes; MAPKi: Mitogen-activated protein kinase inhibitors; MPO: Myeloperoxidase; PD-L1: Anti-programmed cell death protein ligand-1; PD-1: Programmed cell death receptor-1; PET/CT: Positron emission spectrometry/computed tomography; PR3: Proteinase 3 antigen.
\end{abstract}

\section{Acknowledgements}

Not applicable

\section{Authors' contributions}

All the authors contributed to the paper, including writing and revising it. All authors read and approved the final manuscript.

\section{Funding}

The authors received no financial support for the research or for the writing and/or publication of this article.

\section{Availability of data and materials \\ Not applicable}

\section{Declarations}

\section{Ethics approval and consent to participate}

The Ethics Advisory Committee at Baystate Health reviewed the consent documentation, the involvement of human subjects, and the data collection and monitoring in this study and found it to be without ethical violation.

\section{Consent form}

Written informed consent was obtained from the patient for publication of this case report and any accompanying images. A copy of the written consent is available for review by the Editor-in-Chief of this journal.

\section{Competing interests}

The authors declare that they have no competing interests.

\section{Author details}

${ }^{1}$ Dept. of Internal Medicine, University of Massachusetts Medical School -Baystate, Springfield, MA, USA. ${ }^{2}$ Division of Nephrology, University of Massachusetts Medical School -Baystate, Springfield, MA, USA. ${ }^{3}$ Dept. of Pathology, University of Massachusetts Medical School -Baystate, Springfield, MA, USA.

Received: 12 July 2020 Accepted: 1 March 2021

Published online: 03 April 2021

\section{References}

1. Mackiewicz-Wysocka M, Zolnierek J, Wysocki PJ. New therapeutic options in systemic treatment of advanced cutaneous melanoma. Expert Opin Investig Drugs. 2013;22:181-90.

2. Ilieva KM, Correa I, Josephs DH, Karagiannis P, Egbuniwe IU, Cafferkey MJ, et al. Effects of BRAF mutations and BRAF inhibition on immune responses to melanoma. Mol Cancer Ther. 2014;13:2769-83.

3. Yu C, Liu X, Yang J, Zhang M, Jin H, Ma X, et al. Combination of immunotherapy with targeted therapy: theory and practice in metastatic melanoma. Front Immunol. 2019;10:990.

4. Cortazar FB, Marrone KA, Troxell ML, Ralto KM, Hoenig MP, Brahmer $J$ R, et al. Clinicopathological features of acute kidney injury associated with immune checkpoint inhibitors. Kidney Int. 2016:90:638-47.

5. Wei SC, Duffy CR, Allison JP. Fundamental mechanisms of immune checkpoint blockade therapy. Cancer Discov. 2018;8:1069-86.

6. Wanchoo R, Jhaveri KD, Deray G, Launay-Vacher V. Renal effects of BRAF inhibitors: a systematic review by the Cancer and the Kidney International Network. Clin Kidney J. 2016;9:245-51.

7. Perico L, Mandalà M, Schieppati A, Carrara C, Rizzo P, Conti S, et al. BRAF signaling pathway inhibition, podocyte injury, and nephrotic syndrome. Am J Kidney Dis. 2017;70:145-50.

8. Wanchoo R, Karam S, Uppal NN, Barta VS, Deray G, Devoe C, et al. Adverse renal effects of immune checkpoint inhibitors: a narrative review. Am J Nephrol. 2017:45:160-9.

9. McAdoo SP, Pusey CD. Anti-glomerular basement membrane disease. CJASN. 2017:12:1162-72

10. Dammacco F, Battaglia S, Gesualdo L, Racanelli V. Goodpasture's disease: a report of ten cases and a review of the literature. Autoimmun Rev. 2013;12:1101-8.

11. Nasr SH, Collins AB, Alexander MP, Schraith DF, Herrera Hernandez L, Fidler ME, et al. The clinicopathologic characteristics and outcome of atypical anti-glomerular basement membrane nephritis. Kidney Int. 2016:89:897-908.

12. Glassock RJ. Atypical anti-glomerular basement membrane disease: lessons learned. Clin Kidney J. 2016:9:653-6.

13. Sammartino C, Goodman D, Flanagan G, Hill P. Anti-GBM disease following CTLA4 blockade in a patient with metastatic melanoma. Clin Kidney J. 2010;3:135-7.

14. Takahashi N, Tsuji K, Tamiya H, Shinohara T, Kuroda N, Takeuchi E. Goodpasture's disease in a patient with advanced lung cancer treated with nivolumab: an autopsy case report. Lung Cancer. 2018;122:22-4.

15. Wei SC, Levine JH, Cogdill AP, Zhao Y, Anang N, Andrews MC, et al Distinct cellular mechanisms underlie anti-CTLA-4 and anti-PD-1 checkpoint blockade. Cell. 2017;170:1120-33.

16. Simpson TR, Li F, Montalvo-Ortiz W, Sepulveda MA, Bergerhoff K, Arce $F$, et al. Fc-dependent depletion of tumor-infiltrating regulatory $T$ cells co-defines the efficacy of anti-CTLA-4 therapy against melanoma. J Exp Med. 2013;210:1695-710.

17. Selby MJ, Engelhardt JJ, Quigley M, Henning KA, Chen T, Srinivasan $M$, et al. Anti-CTLA-4 antibodies of IgG2a isotype enhance antitumor activity through reduction of intratumoral regulatory $T$ cells. Cancer Immunol Res. 2013;1:32-42.

18. Reynolds J, Tam FWK, Chandraker A, Smith J, Karkar AM, Cross J, et al. CD28-B7 blockade prevents the development of experimental autoimmune glomerulonephritis. J Clin Invest. 2000;105:643-51.

19. Kitching $A R$, Huang $X R$, Ruth A-J, Tipping PG, Holdsworth SR. Effects of CTLA4-Fc on glomerular injury in humorally-mediated glomerulonephritis in BALB/c mice. Clin Exp Immunol. 2002;128:429-35. 
20. Wu J, Hicks J, Borillo J, Glass WF, Lou Y-H. CD4+ T cells specific to a glomerular basement membrane antigen mediate glomerulonephritis. J Clin Invest. 2002;109:517-24.

21. Robertson J, Wu J, Arends J, Zhou C, McMahon J, Torres L, et al. Activation of glomerular basement membrane-specific B cells in the renal draining lymph node after T cell-mediated glomerular injury. JASN. 2005; 16:3256-63.

22. Chaib H, Hoskins BE, Ashraf S, Goyal M, Wiggins RC, Hildebrandt F. Identification of BRAF as a new interactor of PLCE1, the protein mutated in nephrotic syndrome type 3. Am J Physiol Renal Physiol. 2008;294:F93-9.

\section{Publisher's Note}

Springer Nature remains neutral with regard to jurisdictional claims in published maps and institutional affiliations.
Ready to submit your research? Choose BMC and benefit from:

- fast, convenient online submission

- thorough peer review by experienced researchers in your field

- rapid publication on acceptance

- support for research data, including large and complex data types

- gold Open Access which fosters wider collaboration and increased citations

- maximum visibility for your research: over $100 \mathrm{M}$ website views per year

At BMC, research is always in progress.

Learn more biomedcentral.com/submissions 